\section{Malarial infection among antenatal and maternity clinics attendees at the Federal Medical Centre, Makurdi, Benue State, Nigeria}

\author{
Elizabeth Amuta, ${ }^{1}$ Robert Houmsou, ${ }^{2}$ \\ Emmanuel Wama, ${ }^{2}$ Mary Ameh $^{3}$ \\ 'Department of Biological Sciences, \\ University of Agriculture, Makurdi; \\ 2Departement of Biological Sciences, \\ Taraba State University, Jalingo; \\ ${ }^{3}$ Department of Biological Sciences, \\ Benue State University Makurdi, Nigeria
}

\section{Abstract}

This study assessed the level of malarial infection in relation to some epidemiological factors, gravidity and pregnancy period of antenatal clinic attendees of the Federal Medical Centre, Makurdi, Benue State, Nigeria. We also assessed malarial infection in placental blood in relation to gravidity of pregnant women at delivery in the maternity clinic of the same hospital. Thin and thick blood films were prepared for microscopic examination. A questionnaire was administered to each pregnant woman at the antenatal clinic to collect data on educational level, occupation, gravidity, pregnancy period, malaria preventive measures and malaria symptoms. Of the 163 pregnant women examined at the antenatal clinic, $68.3 \%$ (111/163) were infected with malaria. Pregnant women that are illiterates $\left(\chi^{2}=15.44\right.$, $\mathrm{P}=0.100)$ and those that are farmers $\left(\chi^{2}=9.20\right.$, $\mathrm{P}=0.270$ ) had the highest infection rate with no significant difference respectively. Malarial infection was significantly higher in the multigravidae, $57.6 \%(34 / 59) \quad\left(\chi^{2}=5.16, \quad \mathrm{P}=0.007\right)$ and non-significant in the pregnant women at their third trimester of pregnancy, $60.9 \%$ (53/89) $\left(\chi^{2}=4.45, \mathrm{P}=0.108\right)$. Placental malaria was significantly higher in the primigravidae among pregnant women at delivery $\left(\chi^{2}=9.33\right.$, $\mathrm{P}=0.000)$. A significant difference $\left(\chi^{2}=33.52\right.$, $\mathrm{P}=0.000$ ) was observed between pregnant women that did not use any malaria preventive methods, $91.2 \%(31 / 34)$ and those that used single, $64.3 \%$ (65/101) and combined, $46.4 \%$ (13/28) methods of prevention. Malaria remains highly prevalent among antenatal clinics attendees in Makurdi, Nigeria. Combined method of prevention (insecticides treated nets and insecticide spray) yielded good results and its use is advocated in preventing malaria among the pregnant women.

\section{Introduction}

Malaria is a major public health problem in most tropical and subtropical countries where the climate is suitable for the breeding of the Anopheles mosquitoes that transmit the parasite. The spread of the disease depends on socio-economic conditions, population mobility, housing, occupation, human habits and immunity. Its distribution and density vary from place to place.

An estimated 3.3 billion people are at risk for malaria in the world, with sub-Saharan Africa having the highest risk of infection (approximately $80 \%$ of cases and $90 \%$ of deaths worldwide). ${ }^{1}$ Children under five years of age and pregnant women are the most severely affected. An estimated 125 million women become pregnant every year in malaria endemic regions. ${ }^{2}$

Despite the effective launching of the Roll Back Malaria since the year 2001 in most African countries, pregnant women and their unborn babies continue to face a disproportionate high burden of the disease. Pregnant women have a higher risk than the non-pregnant ones to be infected with malaria, and they are at increased risk of adverse events such as anemia, risk of miscarriage, stillbirth, preterm delivery, fetal growth restriction and even death.,

Malaria is endemic throughout Nigeria with an estimated $60 \%$ of the population having at least one to four episodes each year. ${ }^{5} \mathrm{~A}$ Nigerian-based survey showed that $11 \%$ of all maternal deaths and $70.5 \%$ of morbidity during pregnancy are due to malaria. ${ }^{5}$

During the past seven to eight years, the Federal Government of Nigeria through its Ministry of Health intensified the fight against malaria through the distribution of Insecticides Treated Nets and administration of the Intermittent Preventive Treatment (IPT) with Sulfadoxine + Pyrimethamine to the pregnant women attending antenatal clinics in all hospitals across the country. This effort is aimed to reduce by half and reverse the number of malarial infection by 2015 , so that the country can meet up with the Millennium Development Goals. Unfortunately not much has been achieved and malaria still remains a serious threat for pregnant women in Nigeria. Thus, the present study was undertaken to assess epidemiological factors associated with malaria including gravidity and pregnancy period of women attending the antenatal clinic of the Federal Medical Centre, Makurdi, Benue State, Nigeria. The study was also aimed to assess the extent of malarial infection from placental blood of pregnant women at delivery in the maternity clinic of the same hospital in relation to their gravidity.
Correspondence: Elizabeth Amuta, Department of Biological Sciences, University of Agriculture Makurdi, P.M.B 2373 Makurdi, Benue State, Nigeria.

Tel. +23.480 .686 .86900$

E-mail: bettyamuta@gmail.com

Key words: malaria, children, mothers, Benue State, Nigeria.

Acknowledgements: we sincerely thank the management of the Federal Medical Centre, Makurdi for granting us permission to undertake this study. We are grateful to the staff of the antenatal and maternity clinics for their understanding and help rendered during the study. We are also indebted to the pregnant women whose understanding and simplicity towards the study helped in the successful administration of questionnaires and collection of blood samples.

Conference presentation: this paper was presented at the $36^{\text {th }}$ annual conference of the Parasitology and Public Health Society of Nigeria, 2012; Sept 25-28; Federal University of Technology, Akure; Ondo State, Nigeria.

Contributions: EA, RH, conception and study design; MA, EW, collection of samples and literature search; RH, statistical analysis and drafting the manuscript. EA, critically revised the paper.

Conflict of interests: the authors declare no potential conflict of interests.

Received for publication: 29 August 2013.

Revision received: 29 November 2013.

Accepted for publication: 20 December 2013.

This work is licensed under a Creative Commons Attribution NonCommercial 3.0 License (CC BYNC 3.0).

(C) Copyright E. Amuta et al., 2014

Licensee PAGEPress, Italy

Infectious Disease Reports 2014; 6:5050

doi:10.4081/idr.2014.5050

\section{Materials and Methods}

\section{Study area}

The study was undertaken in Makurdi, capital of Benue State, Nigeria. The area is located at latitude $7^{\circ} 43^{\prime} \mathrm{N}$ and longitude $8^{\circ} 32^{\prime} \mathrm{E}$ within the guinea Savannah region of Nigeria. Mean annual temperature ranges from a minimum of $21.7^{\circ} \mathrm{C}$ to a maximum of $35.2^{\circ} \mathrm{C}$ with an annual average rainfall of $1000 \mathrm{~mm}$. There are two distinct seasons, the wet and the dry. The former lasts from April to October, while the latter lasts from November to March. Most dwellers are civil servants, military/police, traders, fishermen and artisans. 


\section{Study population}

Prior to the beginning of the study, ethical approval was obtained from the Ethics Committee of the Federal Medical Centre, Makurdi. Pregnant women who visited the antenatal and maternity clinics of the center between November 2010 and February 2011 were enrolled for the study. All the pregnant women were duly informed on the purpose and significance of the study.

\section{Sample size estimation and study design}

The Statemate 2.00 software was used for sample size estimation and it gave a minimum of 132 for the antenatal by taking proportion from a previous study. The study was observational, cross-sectional. A systematic random sampling was done to enroll the pregnant women that gave their consent. During the 4month period of the study a total of 241 pregnant women were included in the randomization among which we randomly enrolled 192 in the antenatal and 44 during delivery in the maternity clinic. From these, 29 and 23 were excluded respectively from each group because of incomplete information from the questionnaires.

Pregnant women that reported to complain with symptoms such as fever, fatigue, joints pains, diarrhea, nausea and vomiting 4-5 days preceding their visits to the hospital were included in the study, while those that were apparently healthy and did not report any symptoms were excluded.

\section{Questionnaire administration}

A structured questionnaire was administered to each pregnant woman that participated in the study at the antenatal clinic. Data were collected on the literacy level and occupation, malaria preventive measures, pregnancy period, gravidity and malaria symptoms such as headache, cold and chills, loss of appetite, nausea, diarrhea, muscle aches, sweat and fatigue, brown urine and vomiting.

\section{Blood sample collection and labo- ratory examination}

About two millimeters of intravenous and placental blood samples (163 and 21 samples, respectively) were collected into well-labelled EDTA bottles from the pregnant women that were enrolled and taken to the parasitology section of the hospital's laboratory. The thin and thick blood films were prepared from the blood samples collected and stained using 10\% Giemsa as described by Cheesbrough. ${ }^{6}$ The blood films were examined microscopically using oil immersion at X100 objective lens. Two experienced laboratory scientists examined the slides so as to ensure qualitative results.

\section{Data analysis}

Inferential and descriptive statistics were used for data presentation. Data were entered in Microsoft Excel 2007 and imported into SPSS version 17.0 for analysis. Chi-squared test was used to compare malarial infection in pregnant women and their educational level, occupation, gravidity, pregnancy period and preventive methods. The significance level was considered at $\mathrm{P} \leq 0.05$.

\section{Results}

Table 1 shows data on malarial infection in relation to education, occupation, gravidity and pregnancy period of pregnant women attending the antenatal clinic at Federal Medical Centre, Makurdi, Benue State, Nigeria. An overall infection rate of $68.3 \%$ (111/163) was observed among the pregnant women examined. Pregnant women that had no formal education (illiterate) and those that claimed having secondary school level of education had the highest infection rates $(86.7 \%$, $13 / 15)$ and $(81.9 \%, 50 / 61)$ respectively $\left(\chi^{2}=15.44, \mathrm{P}=0.100\right)$. Likewise, pregnant farmers and unemployed pregnant women had the highest malarial infection rates $(86.7 \%, 13 / 15)$ and $(80 \%, 20 / 25)$ respectively $\left(\chi^{2}=9.20\right.$, $\mathrm{P}=0.270$ ). With regards to gravidity, primigravidae, $(71.6 \%, 48 / 67)$ and secundigravidae, (78.4\%, 29/37) had significant higher infection rates than the multigravidae $(57.6 \%, 34 / 59)$ $\left(\chi^{2}=5.16, P=0.007\right)$. Pregnant women at their first, $(75.5 \%, 34 / 45)$ and second, (78.4\%, 29/37) pregnancy trimester had higher infection rates than those at their third trimester, $(60.9 \%, 953 / 87)$ with no significant difference $\left(\chi^{2}=4.45, P=0.108\right)$. Malarial infection in placental blood of pregnant women at delivery in relation to their gravidity showed that primigravidae had a significantly higher malarial infection $(55.5 \%, 5 / 9)$ than the secundigravidae, $(25.0 \%, 1 / 4)$ and multigravidae, $(12.5 \%$, 1/8) $\left(\chi^{2}=9.33, P=0.005\right)$.

Table 2 shows malarial infection rates in

Table 1. Malarial infection in relation to education, occupation, gravidity and pregnancy period of pregnant women attending the antenatal clinic at the Federal Medical Centre, Makurdi, Benue State, Nigeria.

\begin{tabular}{lccc}
\hline Variables & Pregnant women & Malaria infection (\%) & P \\
Education & & & \\
Illiterate & 15 & $13(86.7)$ & 0.100 \\
Primary & 22 & $14(63.7)$ & \\
Secondary & 61 & $50(81.9)$ & \\
Tertiary & 65 & $34(52.3)$ & 0.270 \\
Occupation & & $20(80.0)$ & \\
No occupation & 25 & $48(69.5)$ & \\
Civil/public service & 69 & $30(55.5)$ & \\
Business/Trading & 54 & $13(86.7)$ & \\
Farming & 15 & & \\
Gravidity & & $48(71.6)$ & \\
Primigravidae & 67 & $29(78.9)$ & \\
Secundigravidae & 37 & $34(57.6)$ & \\
Multigravidae & 59 & & \\
Pregnancy period & & $34(75.5)$ & \\
First trimester & 45 & $24(77.4)$ & \\
Second trimester & 31 & $53(60.9)$ & \\
Third trimester & 87 & & \\
\hline
\end{tabular}

Table 2. Malarial infection in relation to preventive methods used among pregnant women attending the antenatal clinic at the Federal Medical Centre, Makurdi, Benue State, Nigeria.

\begin{tabular}{lcc}
\hline Preventive methods & Preginant women & Infected women (\%) \\
None & 34 & $31(91.2)$ \\
Single method & 101 & $65(64.3)$ \\
ITNs & 40 & $17(42.5)$ \\
Repellent cream & 12 & $8(66.6)$ \\
Insecticide spray & 49 & $40(81.6)$ \\
\hline Combined method & 28 & $13(46.4)$ \\
$\quad$ Insecticide + repellent cream & 8 & $7(87.5)$ \\
Insecticide spray + ITNs & 20 & $6(30.0)$ \\
\hline ITNs, insecticide trated nets.
\end{tabular}

ITNs, insecticide treated nets. 
relation to preventive methods used among pregnant women attending the antenatal clinic of Federal Medical Centre, Makurdi, Benue State, Nigeria. A significant difference was observed in malarial infection rate between pregnant women that did not use any malaria preventive methods, $(91.2 \%, 31 / 34)$ and those that used single, $(64.3 \%, 65 / 101)$ and combined, $(46.4 \%, 13 / 28)$ methods of prevention $\left(\chi^{2}=33.52, \mathrm{P}=0.005\right)$. Among pregnant women that used a single method of prevention, those that used Insecticide Treated Nets (ITNs) had lower malaria prevalence $(42.5 \%, 17 / 40)$. Among pregnant women that used combined methods of prevention, those that used insecticide spray + ITNs had lower malaria prevalence $(30.0 \%, 6 / 20)$.

Out of the 163 pregnant women interviewed about knowledge of malaria symptoms, only $15.0 \%$ (25/163) did not know any malaria symptoms and 85.0\% (138/163) knew at least two and more malaria symptoms (Figure 1).

\section{Discussion and Conclusions}

In sub-saharan Africa pregnant women and children under five years of age are the most susceptible and vulnerable individuals to malaria. The high infection rate $(68.3 \%)$ reported in the present study is similar to that found in another study that reported high prevalence (76.9\%) among pregnant women in Gboko, ${ }^{7}$ but higher than that reported in Otukpo (42.3\%), ${ }^{8}$ which are all Local government Areas of Benue State, Nigeria. This high infection rate among the pregnant women in Makurdi could be related to environmental factors such as presence of unclean gutters, insalubrity and residence in swamps which are favorable conditions for the breeding of Anopheles mosquitoes. This high infection rate could be also partly due to the low education, illiteracy and scarce attitudes of the pregnant women towards malaria preventive methods or activities that could expose them to mosquito's bites through their various occupations. The lower malarial infection rate in pregnant women with higher education level is likely related to a better knowledge of preventive measures to avoid this disease. Previous and present data obtained on malaria in Benue State show that malaria still remains a serious public health problem among pregnant women despite the intensified control measures set in place to roll back the disease. Studies reported among pregnant women in other parts of Nigeria found rates of $57.1 \%$ in the NigerDelta; ${ }^{9} 72 \%$ in Osogbo, Osun State; ${ }^{10} 26 \%$ in Port-Harcourt, Rivers State; ${ }^{11} 7.7 \%$ and $52 \%$ in Lagos State. ${ }^{12,13}$ When compared to other subSaharan countries endemic for malaria, malarial infection rate among pregnant women in

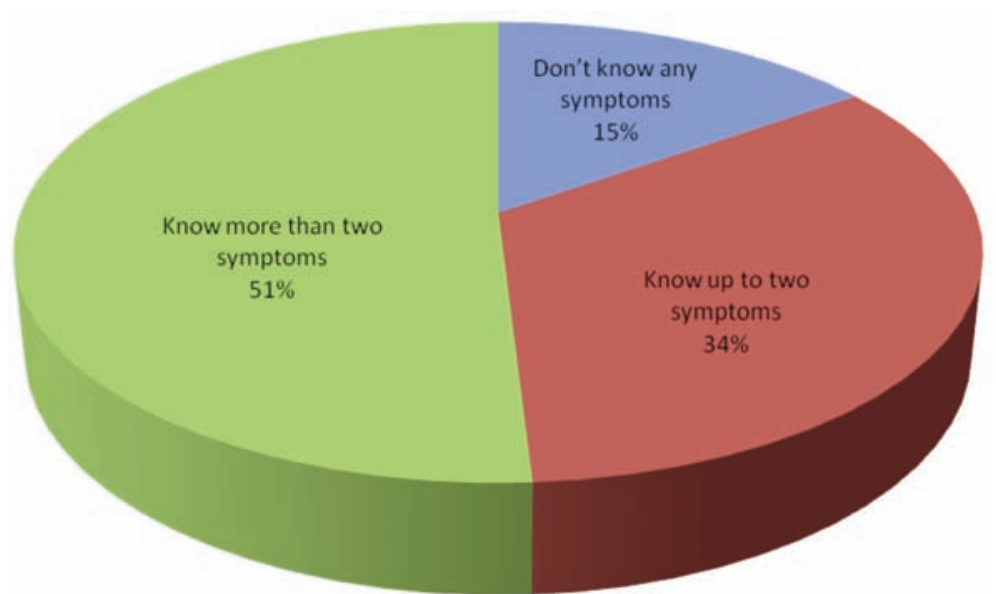

Figure 1. Knowledge of malaria symptoms among pregnant women attending antenatal services at Federal Medical Centre, Makurdi, Benue State, Nigeria.

the present study is higher than $40.4 \%, 26.9 \%$ and $18 \%$ reported in Cameroon, ${ }^{14}$ Mozambique, ${ }^{15}$ Kenya, ${ }^{16}$ respectively.

In this study, primi- and secundigravidae women were more infected than multigravidae. This could be due to poor knowledge on the effect of exposure to mosquitoes' bites during their pregnancy period in contrast to the multigravidae who are likely more familiar with health management of preventive methods and control measures against malaria. This is also likely due to past information on prevention measures in their previous pregnancy when attending the antenatal clinics, even though no data are available regarding this hypothesis. Multigravidae would have also developed more immunity to malaria during pregnancy than the primi-and secundigravidae that are just at their first or second pregnancy. The low prevalence in the multigravidae could be also due to the inhibition of type 1 cytokine responses (interferon, interleukin 2 and 12) known as the factor responsible for the susceptibility of primigravidae to malaria infection. ${ }^{17}$ Our findings are in agreement with those of other several studies that reported primigravidae to be more exposed to malaria than multigravidae. ${ }^{6-12,18}$

The high malarial infection rate in pregnant women at their first and second trimester of pregnancy corroborates the findings which reported that malaria peaks during the 13-17 weeks of pregnancy and declines towards the third trimester, ${ }^{19}$ even though there are equivocal data from studies conducted in Lagos, Nigeria and Garoua, Cameroon that found higher infection rates in the third trimester of pregnancy. ${ }^{12,13}$ Variations in malarial infection during the different trimesters of pregnancy could be due to some prevailing factors like the immuno-genetic constitution of the pregnant women, exposure to mosquito bites and resi- dence in stable or unstable malaria transmission areas. With regards to placental malaria, $33.3 \%$ of the pregnant women at delivery were observed to be infected; this confirms findings by Dessai et al. ${ }^{20}$ who reported a $26.0 \%$ rate in sub-Saharan Africa. The present study reports that primi- and secundigravidae at delivery were more infected with malaria than the multigravidae. This is likely due to the lack of immunity to pregnancy-specific variants of Plasmodium falciparum found in multigravidae whose immunity to malaria is acquired over subsequent pregnancies. As a result they can develop antibodies to prevent $P$. falciparum sequestration and enhance opsonic clearance of parasitized erythrocytes. ${ }^{21-24}$

The present study shows that preventive methods are variously effective, but the combination of ITNs + Insecticide spray seemed to be more effective than the use of insecticide spray or repellant cream alone. The reported good result of this combination of preventive methods could just reflect a synergy between the chemical components against mosquitoes. The high malarial infection rate observed among pregnant women that used insecticide sprays or repellant cream alone, and even combination of these two methods could be due to mosquitoes resistance to the chemical or to the low quality of the insecticides sold; finally, the short duration of the repellant cream which lasts for about five to six hours only could be another important issue. Combination of preventive methods against malaria also yielded good results in Garoua, Cameroon where Hile et al..$^{14}$ reported no single infection among pregnant women that used Insecticide spray + ITNs.

The cross-sectional design of this study is a major limitation that did not permit us to obtain data on the treatment follow-up, and on the effect of malaria on the newborn outcome, 
abortion, premature delivery and low-birth weight.

The present study reveals that malaria remains highly prevalent among pregnant women attending antenatal clinics of the Federal Medical Centre, Makurdi, Benue State, Nigeria, despite the initiative taken more than a decade ago to roll it back. Combined method of prevention (ITNs + Insecticide spray) yielded good results in preventing malaria among the pregnant women in Makurdi. It is therefore recommended that: i) Intermittent Screening and Treatment (IST) which is an alternative clinical management approach based on active case detection and immediate treatment of malaria could be adopted in sub-Saharan Africa, as it has yielded good results and is being currently implemented in many parts of Asia. Pregnant women should be advocated during their antenatal visits to use combination of preventive methods like Insecticide spray + ITNs so as to reduce the risk of Anopheles mosquitoes' bites; ii) pregnant women at their first antenatal visits should be given free ITNs. This is to avoid mosquitoes' bites during their early pregnancy; iii) jingles on malaria preventive/control measures should be done in as many languages as possible using the radio, TV, local rallies, etc.

\section{References}

1. WHO. World malaria report 2012. Available from: http://apps.who.int/iris/bitstream/ 10665/78945/1/9789241564533_eng.pdf?ua $=1$

2. Dellicour S, Tatem AJ, Guerra CA, et al. Quantifying the number of pregnancies at risk of malaria in 2007: a demographic study. PLoS Med 2010;7:e1000221.

3. Brabin BJ. An analysis of malaria in pregnancy in Africa. Bull World Hlth Org 1983;61:1005-16.

4. McGregor IA, Wilson ME, Billewicz WZ. Malaria infection of the placenta in the
Gambia, West Africa; its incidence and relationship to stillbirth, birth weight and placental weight. Trans R Soc Trop Med Hyg 1983;77:232-44.

5. Federal Ministry of Health. Malaria during pregnancy: in the context of focused antenatal care. An orientation package for health care providers copyright. Abuja: FMoH and Malaria Action Coalition; 2004. pp 66-71.

6. Cheesbrough, M. Medical laboratory manual for countries part 2. 2nd ed. Cambridge: University Press; 2000.

7. Houmsou RS, Amuta EU, Sar TT, et al. Malarial infection in pregnant women attending antenatal clinics in Gboko, Benue State, Nigeria. Int $\mathrm{J}$ Acad Res 2010;2:33-6.

8. Jombo GTA, Mbaawuaga EM, Ayegba AS, et al. How far have we rolled back malaria on the African continent nine years down? The burden of malaria among pregnant women in a semi-urban community of northern Nigeria. J Medicine Med Sci 2010;1:235-41.

9. Chukwuocha UM, Dozie IN, Chukwuocha AN. Malaria and its burden among pregnant women in parts of the Niger Delta area of Nigeria. Asian Pac J Reprod 2012;1:147-51.

10. Adefioye, OA, Adeyeba OA, Hassan WO, et al. Prevalence of malaria parasite infection among pregnant women in Osogbo, Southwest, Nigeria. Am Eur J Sci Res 2007;21:43-5.

11. Wogu, MN, Nduka FO, Wogu MD. Prevalence of malaria parasite infection among pregnant women attending antenatal clinics in Port Harcourt, Rivers State, Nigeria. Int J Trop Dis Hlth 2013;3:126-32.

12. Agomo C0, Oyibo WA, Anorlu RI, et al. Prevalence of malaria in pregnant women in Lagos, South-West Nigeria. Kor J Parasitol 2009;47:179-83.

13. Raimi OG, Kanu CP. The prevalence of malaria infection in pregnant women living in a suburb of Lagos, Nigeria. Afr J
Biochem Res 2010;4 243-5.

14. Hile T, Assam Assam JP, Amali 0, et al. The epidemiology of malaria among pregnant women in Garoua, Northern Cameroon. J Parasitol Vect Biol 2012;5:1-5.

15. Bardají A, Sigauque B, Bruni L, et al. Clinical malaria in African pregnant women. Mal J 2008;7:27.

16. Ouma P, van Eijk MA, Hamel MJ, et al. Malaria and anaemia among pregnant women at first antenatal clinic visit in Kisumu, western Kenya. Trop Med Int Health 2007;12:1515-23.

17. McGregor LA. Epidemiology of malaria and pregnancy. Am J Top Med Hyg 2010;33:51725.

18. Guyatt HL, Snow RW. Impact of malaria during pregnancy on low birth weight in Sub-saharan Africa. Clin Microbiol Rev 2004;17:760-9.

19. Falade CO, Tongo 00, Ogunkunle 00, et al. Effects of malaria in pregnancy on newborn anthropometry. J Infect Dev Ctries 2010;4:448-53.

20. Dessai M, ter Kuile F0, Nosten F et al. Epidemiology and burden of malaria in pregnancy. Lancet Infect Dis 2007;7:93104.

21. Fried M, Nosten F, Brockman A, et al. Maternal antibodies block malaria. Nature 1998;395:851-2.

22. Ricke CH, Staalsoe T, Koram K, et al. Plasma antibodies from malaria-exposed pregnant women recognize variant surface antigens on Plasmodium falciparuminfected erythrocytes in a parity-dependent manner and block parasite adhesion to chondroitin sulfate A. J Immunol 2000;165: 3309-16.

23. Keen J, Serghides L, Ayi K et al. HIV impairs opsonic phagocytic clearance of pregnancy-associated malaria parasites. PLoS Med 2007;4:e181.

24. Andrea L, Conroy, Chloe R, et al. Malaria in pregnancy: diagnosing infection and identifying foetal risk. Expert Rev Anti Infect Ther 2012;10:1331-42. 